\title{
Part 14: Platform Speeches
}

\author{
Bridging the Gap Between Research and Practice: \\ Investigation of Needs and Characteristics of End- \\ users, for a Future Inclusion of BCIs in AT-centers \\ Riccio Angela ${ }^{\mathrm{a}, *}$, Schettini Francesca ${ }^{\mathrm{b}}$, Giraldi Enri- \\ $\mathrm{co}^{\mathrm{a}}$, Cincotti Febo ${ }^{\mathrm{a}, \mathrm{c}}$ and Mattia Donatella ${ }^{\mathrm{a}}$ \\ ${ }^{a}$ Neuroelectrical Imaging and BCI Lab, IRCCS Fon- \\ dazione Santa Lucia, Rome, Italy \\ ${ }^{\mathrm{b}}$ Servizio Ausilioteca per Riabilitazione Assistita con \\ Tecnologia (SARA-t), Fondazione Santa Lucia (IR- \\ CCS), Rome, Italy \\ ${ }^{\mathrm{c}}$ Department of Computer, Control, and Management \\ Engineering Antonio Ruberti, "Sapienza" University \\ of Rome, Rome, Italy
}

Background: Brain computer interfaces (BCIs) measure signals related to specific brain activity and translate them into outputs to control external devices. It is demonstrated that $\mathrm{BCI}$ can provide people with communication disorders with an Assistive Technology (AT) restoring their interaction with the environment. Despite BCI technology could improve inclusiveness of AT solutions, BCIs are not currently available in the AT-centers portfolio. A step forward the inclusion of BCIs in AT-centers, is their integration with existing (available in the market) assistive or mainstream technologies, resulting in a hybrid BCI-based communication device. The aim of the study is to investigate the characteristics of patients attending an AT-center, who could take advantage from BCI introduction in AT-centers as an additional/alternative AT channel. We believe that a step to bridge the translational gap between BCI development and end-users, consists in the clear definition of users' characteristics and their ability to control a BCI.

Method: Ten patients ( $43.3 \pm 9.9$ y.o., 2 men) with different diagnosis participated in the study. All patients had undergone a multidisciplinary evaluation and an AT training in an AT-center, because they were limited in (at least) one aspect related to interpersonal communication and/or interaction with digital technologies. Protocol consists of two part . i) Need assessment: pa- tients were administered with the Individual Prioritized Problems Assessment (IPPA) to investigate their needs and they were involved in an AT training aimed at identifying the AT solution matching them. Patients agreed to participate in the BCI session, which will be performed in the next weeks; $i$ i) BCI session: scalp potential will be acquired by means of 16 active electrodes. A P3-speller interface (a matrix of alphabetic items randomly flashing) will be displayed. Patients will be asked to spell words by focusing attention on the desired items. The Tobii Technology $4 \mathrm{C}$ eye-tracker will be used to collect eye-gaze data.

Key results: Fifty-eight problems in total were identified, with an average of 6 (5.8) problems for participant $(\mathrm{SD}= \pm 1.9 ; \min =1$, $\max =7)$. Problems most commonly reported concerned "reading/writing" ( $\mathrm{N}=8$ patients), "communication" ( $\mathrm{N}=7$ patients) and "phone access" ( $\mathrm{N}=6$ patients). AT solutions included touch screen $(\mathrm{N}=4)$, head tracker $(\mathrm{N}=2)$ and eye tracker $(\mathrm{N}=3)$ as input channels to control customized user interfaces. Relationship between patients' clinical and neurophysiologic (ERPs) characteristics and BCI control performance will be reported. Influence of eye-movements will also be reported.

Conclusion: In this study, we reported preliminary data about needs and matching AT solutions of potential end-users of a hybrid-BCI device for communication. The overall aim is to generate profiles of patients that would potentially use the BCI as an additional/alternative channel for AT. With this aim, in the next step, patients' performance in controlling a P3based BCI will be investigated and the relationship with user's characteristics (clinical and neurophysiological) will be established. We consider this as an important step for the integration of BCI with daily AT devices (personalized hybrid $\mathrm{BCI}$ ) and for $\mathrm{BCI}$ inclusion in the AT-centers portfolio.

Keywords: Brain-computer interface, communication, users' need, hybrid, eye-tracker.

Acknowledgement: This work was partially supported by the Promobilia Foundation (Grant Ref. 17137). 
*Corresponding author. a.riccio@ hsantalucia.it

\author{
Environment and People Perceptions: The Experi- \\ ence of NEVArt, Neuroestethics of the Art Vision \\ M. Coccagna ${ }^{\mathrm{a}, *}$, P. Avanzini ${ }^{\mathrm{b}}$, M. Fabbri Destro ${ }^{\mathrm{b}}$, G. \\ Vecchiato $^{\mathrm{b}}$, A. Banzi ${ }^{\mathrm{c}}$, R. Folgieric ${ }^{\mathrm{c}}$, V.A. Sironi ${ }^{\mathrm{c}}$ and \\ S. Mazzacane ${ }^{\mathrm{a}}$ \\ ${ }^{\mathrm{a}}$ CIAS (Research Centre for pollution control in high \\ sterile rooms), Department of Architecture, University \\ of Ferrara, cias@unife.it, via della Ghiara 36, 44121 \\ Ferrara, Italy \\ ${ }^{\mathrm{b}}$ Institute of Neuroscience, National Research Council \\ of Italy, Parma, Italy \\ ${ }^{\mathrm{c}}$ CESPEB (Research Institute on the History of Biom- \\ edical Thought), Milan, Italy
}

Background: For several years, neuroscience has been involved in understanding the neurobiological bases of how people perceive their space and every common activity and to change organizational and architectural tools, to brake the existing barrier for a universal designed environment. Dues to the difficulties in using medical devices outside laboratories, scientists simulated their scenarios. The obtained results followed the classical view of cognitive science, which considers the brain as a machine that processes stimuli in a hierarchical way, at different stages of complexity. The chance of conducting real field studies is now easier through the increasing availability of low size sensors and wireless networks for EEG and EMG analysis. The same for other physical and physiological parameters, such as the tracking of eye movements and the electrical conductivity of the skin, closely related to the individual's most instinctive and unconscious reactions.

Method: The NEVArt project wants to investigate a typical emotional feature, as the evaluation of the artistic production and the wide aesthetic involvement generated by the vision of an art work, using a scientific approach and a statistically relevant amount of data. The research aims also to compare and evaluate both the medical and signal affordability of data generates by new Wi-Fi tools, often developed outside the health field (i.e. video games, meditation skills, mental-physical sport performance, etc). The first research step is to analyse how the brain perceive emotions in real scenarios, to further exploit this information improving the architectural environment, the way of exhibiting art and the approach to people that, dues to their specific background or physical-mental capacities, perceive some elements as a barrier or an im- provement (to understand or to be understood). The NEVArt research group (including neurologists, engineers, architects, psychologists, ICT and art experts) will detect the aforementioned parameters by monitoring volunteer (at least 600 people) to analyse their explicit and implicit reactions using different kind of sensors, during their visit to the exhibition "Painting affections: sacred painting in Ferrara between the "500 and the '600"', set up at the Estense Castle in Ferrara from 26 January to 26 December 2019. The study database include: records collected by the investigator upstream (crowding, thermos-hygrometric context, lighting, noise, etc.), non-sensitive and anonymous data filled in by the voluntary visitors in the first section of a questionnaire (age, schooling, habit of attending exhibitions, interest in the subject, level of fatigue/initial fatigue, etc.); subjective evaluation by the volunteer of the works viewed (in the second section of the survey); the tracks of each analysis tool worn (including the video made in real time with the eye tracker). All data will be uploaded at the end of each working day by the investigator, within a protected database (created by CIAS and already used for other similar clinical surveys). All the recorded materials will be periodically analysed and statistically reelaborated using T-test and ANOVA, by the research group, relating objective data (collected with sensors) and objective data (entered by the participant and the experimenter), so as to assess the incidence of elements of agreement and/or dissonance.

Key results: NEVArt may provide a framework of people's physiological, neurological and cognitive emotional reactions, on a broad statistical basis, during the vision of art (now painting but following sculpture, architecture, etc.). The main NEVArt aims are:

1. obtain a neurobiological validation of the experience of aesthetic perception, with particular reference to bodily and mental involvement (neurovegetative, motor, emotional), both implicit (from EEG, galvanic) and explicit (from survey), facing the artistic work and testing the possibility of calibrating some of these sensory levels (disgust, indifference, pleasantness, etc.);

2. quantify the intensity and type of instrumental signals recorded as a function of the level of aesthetic perception, comparing the obtained results through multisensory EEG, EMG and eyetracking tools in real museum conditions (Scenario A) and with high sensitivity EEG 64 channels sensors, EMG and eyetracking for a limited number of paintings and using a low environmental stimulation (Scenario B); 
3. to identify the descriptive parameters of the cited experiences, in order to create a significant scale to represent the physical, emotional, cognitive responses on external strains, referable to the art vision.

Conclusion: Due to the CIAS experience on large clinical surveys, the collection of a huge amount of data it's extremely useful to consolidate the information collected through different sensory channels and diverse tools. Using this method, it will be possible to create a stable framework of information within study reactions, comparing these background data to similar stimuli by people with specific disabilities. Otherwise, the risk of lack of reliability of records, may confuse the perceptions based on experience and knowledge of people (which is expected to have common variations among all participants) with those one arising from cognitive or sensory deficits. The analysis of a feeling perceptions as the "aesthetic pleasure", it is well suited to identify a set of primordial elements that can be tested and stimulated even in subjects with different abilities. The research knowledge, both in term of technical skills and upgrade in neuroscientific awareness, will be fundamental to proceed with a set of new research topics. These expected improvement (partly implemented in parallel with the second stage of NEVArt) include the sense of smell (i.e. product and environmental odours), the didactic field (teaching methodologies aware of vision and perception features), medical purposes, in particular improving some past research of the CIAS about the Parkinson's disease and some new studies about the communication with people with specific cognitive disorders, as Autism, in order to relate their emotional/physiological/cognitive response to different external stresses (starting from basic stimuli as colour, forms, lights, smell, etc.). Expected results also on the exhibit field, to provide suggestions on how to achieve the best way to display art works, enhancing the elements with the greatest impact on visitors. The environmental field, improving new methods to measure the subjective response of users to setting parameters (light, heat, cold, etc.) in order to combine the analysis of objective standards and on the field perceptions. In the safety topics, studying the impact of sensory data to verify the users' perceptions in emergency situations and how to take into account people awareness in emergency preparedness, especially with reference to wayfinding and to use of signs, lights and colours.

Keywords: neuroestethics, art, beauty, perceptions *Corresponding author. E-mail: cnm@unife.it 